\title{
Therapeutic penetrating keratoplasty for the treatment of microbial keratitis due to Staphylococcus MRSA
}

\author{
Panagiota Petrakogianni, Ioanni A. Mallias(D) \\ Laser Plus Eye, Nea Smyrni, Athens, Greece
}

\begin{abstract}
This is a case report of one patient who was diagnosed with microbial keratitis caused by staphylococcus MRSA. Its characteristics, etiology and symptoms will be described below. Moreover, we will discuss the clinical features, treatment plan and treatment outcome on our patient.
\end{abstract}

KEY WORDS: microbial keratitis; penetrating keratoplasty; staph MRSA

Ophthalmol J 2019; Vol. 4, 40-43

\section{INTRODUCTION}

Methicillin-resistant Staphylococcus aureus (MRSA) is a form of contagious bacterial infection that is resistant to numerous antibiotics including methicillin, amoxicillin, penicillin, and oxacillin. This resistance makes it challenging to treat [1]. Methicillin is an antibiotic related to penicillin; it was once effective against staphylococci (staph), a type of bacteria. Staph bacteria have since developed a resistance to penicillin-related antibiotics, including methicillin. These resistant bacteria are called methicillin-resistant staphylococcus aureus, or MRSA. If staph MRSA is diagnosed, its treatment will vary depending on a lot of factors, such as the type of infection, the location of infection, the severity of symptoms and the antibiotics to which the strain of MRSA responds [2]. Medication options for MRSA skin and soft tissue infections may include clindamycin, doxycycline, minocycline, trimethoprim and sulfamethoxazole; rifampin and linezolid are treatment options for MRSA skin and soft tissue infections. Staphylococcus aureus (MRSA) may severely infect the eye including the cornea, the anterior chamber and the vitreous body. Microbial keratitis and endophthalmitis due to staph MRSA are vision-threatening infections and if not treated properly may result in severe loss of visual acuity or even blindness [3]. A case series of catastrophic eye infections caused by MRSA has been reported recently in patients after Lasik and cataract surgery [4-7]. Microbial keratitis if not treated appropriately may lead to cornea perforation and endophthalmitis.

\section{CASE REPORT}

A 72-year-old patient, who was functionally one-eyed, contacted us reporting redness and foreign body sensation in his left eye. As he lives on an island far away from our clinic, it was highly unlikely that he would come to the clinic soon enough. Thus, with the view to assisting him in the best possible way we prescribed moxifloxacin and urged him to contact an ophthalmologist on his island. The patient did not trust the local ophthalmologist's diagnosis; therefore, he preferred to come and be examined in our clinic. 


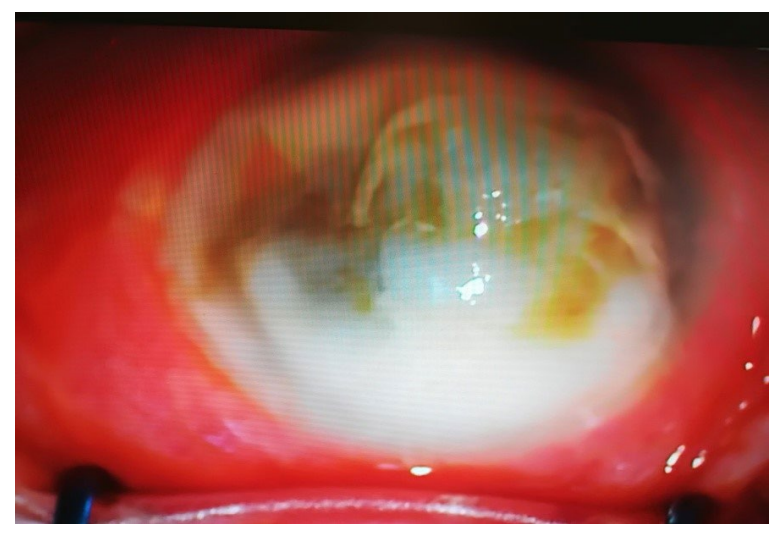

FIGURE 1. The photograph of the eye we took just before the surgery of penetrating keratoplasty

The case-history of this patient was that he had lost vision in his right eye in the past due to an unsuccessful surgery for the detachment of the retina. As he also suffered from type 2 diabetes, he had an inflammation in macula in his left eye. Thus, we administered injections with anti-VEGF factors on a regular basis. Moreover, he used a lens in his left eye, as the eye was aphakic after endocapsular cataract surgery many years ago. His visual acuity was NLP for the right eye and 20/40 for his left eye. In the past month, the patient had been treated with Simbrinza, Duotrav and Acetazolamide pills, as he had also developed increased eye pressure. The examination was indeed conducted four days later. The examination led to an urgent penetrating keratoplasty due to an extensive infection in the cornea. The patient was prescribed moxifloxacin to be used every hour one day before the surgery (Fig. 1). The next day penetrating keratoplasty was performed. During the surgery a terphination of $8.5 \mathrm{~mm}$ was used because the patient was aphakic. During the surgery, wash out of the anterior chamber was performed combined with limited anterior vitrectomy. Intraoperatively, we saw a grey mash on the posterior segment of the eye (Fig. 2) which was due to choroidal detachment caused by severe inflammation. The host cornea was sent to microbiology lab. During the first post-operating days, the patient was administered antibiotics that covered both gram positive (moxifloxacin) and gram negative bacteria (amikacin fortified drops) as well as fungi (voriconazole drops and orally). The results of the microbiological lab tests were negative, so we decided to send the lens and the lenses' case he had used for

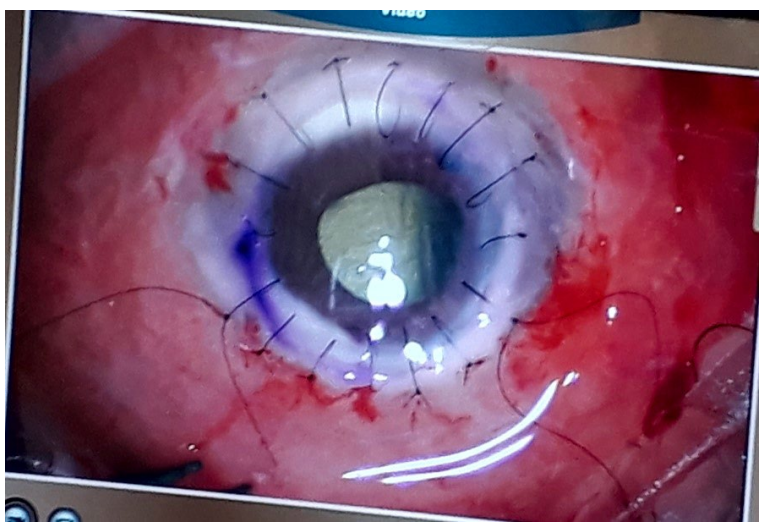

FIGURE 2. The image we took after the surgery of penetrating keratoplasty was over

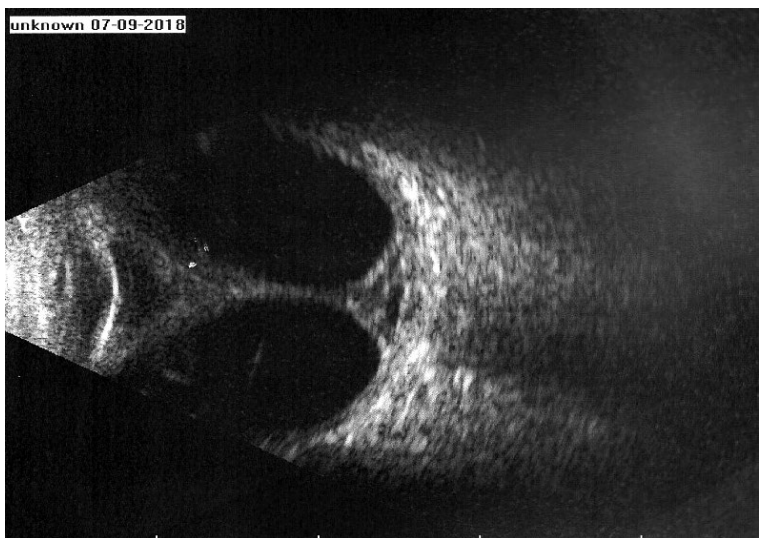

FIGURE 3. Choroidal detachment (Kissing choroidals)

the correction of aphakia for PCR. The surgery of the penetrating keratoplasty was successful. The patient was treated with both moxifloxacin and amikacin fortified drops, as we did not know what had originally caused the inflammation. We stopped this particular treatment when the microbiological laboratory where we had sent the lens informed us that they had found staphylococcus MRSA. His visual acuity for the next month was LP. One week after the surgery, the intraocular pressure was still under $10 \mathrm{~mm} \mathrm{Hg}$, and after a B-scanning we noticed that the inflammation had infected the pars plana, as the patient had a choroidal detachment due to severe inflammatory reaction to microbial keratitis (Fig. 3).

We decided to increase the dose of methylprednisolone we had already prescribed and to examine our patient once a week. One month later, the visual acuity of his left eye was $1 / 20$. Through a B-scanning examination, we saw that the serous choroidal detachment had already been cured (Fig. 4). 


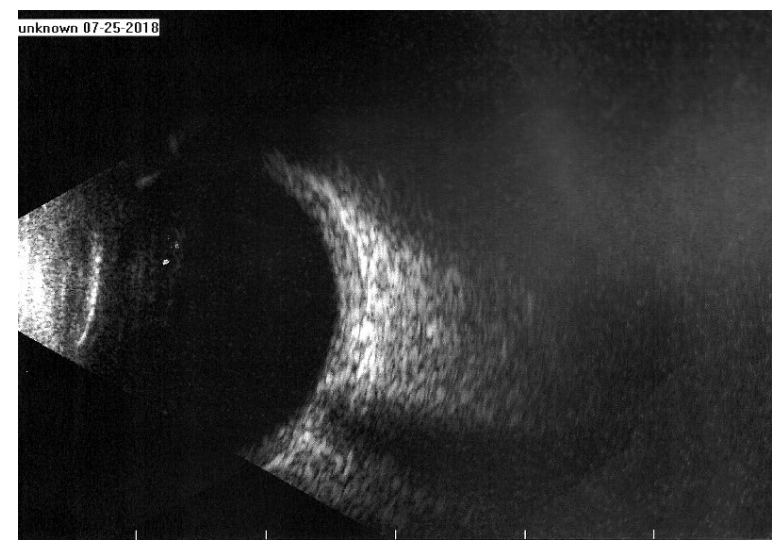

FIGURE 4. B-scanning image a month after the surgery. The choroid is totally cured

It has been six months since the operation was performed. Now, the patient has a visual acuity $1 / 20$ on his left eye and he is only using moxifloxacin on a regular basis.

\section{DISCUSSION}

When the microbiological tests on the graft came as negative on account of moxifloxacin, we went on to send the lenses' case for PCR, which turned out to be positive for MRSA. Staphylococcus MRSA may bring on severe microbial keratitis. It's remarkable that during the first attempt to detect which microbe had caused the inflammation, the answers did not include Staphylococcus MRSA. Prior to the operation, there was no Bscan in order to detect the choroidal detachment. The choroidal detachment was cured after the increased dose of corticosteroids and moxifloxacin [8-10]. We treated these symptoms with Medrol in advance, and they were cured within twenty-five days. As a result, we have come to the conclusion that, regarding the choroidal detachment, it is preferable to insist on the administration of cortisone before we proceed to an operation. [11] There was resistance of the Staphylococcus MRSA to moxifloxacin or vancomycin.

Community-associated MRSA (CA-MRSA) is becoming increasingly prevalent, and ophthalmologists will see more ophthalmic MRSA infections. Although ophthalmic CA-MRSA commonly presents as preseptal lid infection and conjunctivitis, sight-threatening infections also occur. Ophthalmologists must identify MRSA patients, adjust empirical treatment regimens where MRSA is endemic, and take steps to control emergence of resistant or- ganisms in both inpatient and outpatient practices $[12,13]$. Three of the five patients with MRSA keratitis had nosocomial infection. In all but one, effective antibiotic coverage was initiated empirically. That patient had atopic dermatitis with a history of shield ulcers bilaterally and was administered ciprofloxacin ophthalmic drops as therapy initiation. The isolate proved resistant to both levofloxacin and ciprofloxacin. Of the other patients, two had a history of cocaine use (one with definite crack keratopathy), one had preceding herpes zoster ophthalmicus after decompressive craniotomy for intraparenchymal hematoma due to motor vehicle collision, and one suffered trauma to the eye when a container of medical waste exploded. The latter patient was considered to have nosocomial infection, and the MRSA isolate had reduced susceptibility to levofloxacin and resistance to tetracycline [14-16].

\section{REFERENCES}

1. Nichols H. All you need to know about MRSA. Medical News Today. https://www.medicalnewstoday.com/articles/10634.php.

2. Deresinski S. Methicillin-resistant Staphylococcus aureus: an evolutionary, epidemiologic, and therapeutic odyssey. Clin Infect Dis. 2005; 40(4): 562-573, doi: 10.1086/427701, indexed in Pubmed: 15712079.

3. O' Callaghan RJ. The Pathogenesis of Staphylococcus aureus Eye Infections. Pathogens. 2018; 7(1): pii: E9.-134, doi: 10.3390/pathogens7010009, indexed in Pubmed: 29320451.

4. Solomon R, Donnenfeld ED, Perry HD, et al. Methicillin-resistant Staphylococcus aureus infectious keratitis following refractive surgery. Am J Ophthalmol. 2007; 143(4): 629-634, doi: 10.1016/j.ajo.2006.12.029, indexed in Pubmed: 17320811.

5. Deramo VA, Lai JC, Vinokur J. Visual outcome and bacterial sensitivity after methicillin-resistant Staphylococcus aureus-associated acute endophthalmitis. Am J Ophthalmol. 2008; 145(3): 413-417, doi: 10.1016/j.ajo.2007.10.020, indexed in Pubmed: 18191097.

6. Major JC, Engelbert M, Flynn HW. Staphylococcus aureus endophthalmitis: antibiotic susceptibilities, methicillin resistance, and clinical outcomes. Am J Ophthalmol. 2010; 149(2): 278-283, doi: 10.1016/j. ajo.2009.08.023, indexed in Pubmed: 19926069.

7. McCulley JP, Shine WE. Meibomian gland function and the tear lipid layer. Ocul Surf. 2003; 1(3): 97-106, indexed in Pubmed: 17075642.

8. Capper SA, Leopold IH. Mechanism of Serous Choroidal Detachment. AMA. Archives of Ophthalmology. 1956; 55(1): 101-113, doi: 10.1001/ archopht.1956.00930030103014, indexed in Pubmed: 13275180.

9. Chin E. Choroidal Detachment: Serous, Appositional/Kissing. EyeRounds. https://webeye.ophth.uiowa.edu/eyeforum/atlas/pages/ choroidal-detachments/SAK.htm.

10. Reddy AC, Salim S. Choroidal Effusions. EyeNet. American Academy of Ophthalmology. (https://www.aao.org/eyenet/article/choroidaleffusions).

11. Moster MR. How to manage choroidal detachment. Ophthalmology Times. https://www.ophthalmologytimes.com/glaucoma/howmanage-choroidal-detachment.

12. Kirby WM. Extraction of a high potent penicillin inactivator from penicillin resistant Staphylococci. Science. 1944; 99(2579): 452.-453, doi: 10.1126/science.99.2579.452, indexed in Pubmed: 17798398.

13. Rozwadowska-Dowzenko M, Afek-Kamińska M, Wiśniewski J, et al. [Infection with penicillin resistant staphylococci]. Med Dosw Mikrobiol. 1950; 2(2): 183-184, indexed in Pubmed: 14815307.

14. Fattom Al, Sarwar J, Ortiz A, et al. A Staphylococcus aureus capsular polysaccharide (CP) vaccine and CP-specific antibodies protect mice against bacterial challenge. Infect Immun. 1996; 64(5): 1659-1665, indexed in Pubmed: 8613375. 
15. Lee JC, Park JS, Shepherd S, et al. Protective efficacy of antibodies to the Staphylococcus aureus type 5 capsular polysaccharide in a modified model of endocarditis in rats. Infect Immun. 1997; 65(10): 4146-4151., indexed in Pubmed: 9317020.

16. Hiramatsu K, Aritaka N, Hanaki H, et al. Dissemination in Japanese hospitals of strains of Staphylococcus aureus heterogeneously resist- ant to vancomycin. Lancet. 1997; 350(9092): 1670-1673., indexed in Pubmed: 9400512

17. Shanmuganathan VA, Armstrong M, Buller $A$, et al. External ocular infections due to methicillin-resistant Staphylococcus aureus (MRSA). Eye (Lond). 2005; 19(3): 284-291, doi: 10.1038/sj.eye.6701465, indexed in Pubmed: 15375372. 